Tropical Journal of Pharmaceutical Research December 2015; 14 (12): 2179-2185

ISSN: $1596-5996$ (print); 1596-9827 (electronic)

(C) Pharmacotherapy Group, Faculty of Pharmacy, University of Benin, Benin City, 300001 Nigeria.

All rights reserved.

Available online at http://www.tjpr.org

Original Research Article

http://dx.doi.org/10.4314/tjpr.v14i12.4

\title{
TGF- $\beta 1$ and IL-10 expression in epithelial ovarian cancer cell line A2780
}

\author{
Xin Feng ${ }^{1,2 \star}$, Cai-Xia Wang ${ }^{3}$ and Zhi-Ying $\mathrm{Ou}^{4}$ \\ ${ }^{1}$ Department of Gynecologic, Oncology Cancer Center Southern Medical University, Guangzhou, China; ${ }^{2}$ Department of \\ Gynecologic, Guangzhou Women and Children's Medical Center, Guangzhou Medical College, Guangzhou, China; \\ ${ }^{3}$ Department of Gynecology and Obstetrics, Guangdong Provincial Corps Hospital, Chinese People Armed Forces, \\ Guangzhou 51507, China; ${ }^{4}$ Guangzhou Women and Children's Medical Center, Affiliate of Guangzhou Medical College, \\ Guangzhou 510623, China
}

${ }^{*}$ For correspondence: Email: fengxin142@gmail.com; Tel/Fax: 0086-20-61640114

Received: 20 July 2015

Revised accepted: 24 October 2015

\begin{abstract}
Purpose: Ovarian cancer is a leading cause of death among gynaecological malignancies. Transforming growth factor-beta 1 (TGF- $\beta 1$ ) and interleukin-10 (IL-10) are cytokines in the tumour microenvironment and may play critical roles in immune suppression. This study highlights these roles and immunosuppressive functions in epithelial ovarian cancer (EOC).

Methods: TGF- $\beta 1$ and IL-10 expression was compared in malignant, benign, and borderline cancerous tissues and tumour-free tissue by immunohistochemistry. Relationships among the levels of these cytokines, correlation of expression level with EOC prognosis, and cytokine involvement in immunosuppression were investigated.

Results: Immunohistochemical analysis of TGF- $\beta 1$ and IL-10 in epithelial cells showed the presence of epithelial, borderline, and benign ovarian tumour growth, and normal ovarian growth. TGF- $\beta 1$ ( $P=$ 0.121), residual tumour after surgery $(P=0.231)$ and standard chemotherapy $(P=0.121)$ were prognostic factors for EOC. There were no significant differences in clinicopathologic factors between specimens expressing TGF- $\beta 1$ at low and high levels, indicating that TGF- $\beta 1$ is an independent factor in EOC diagnosis. Higher concentrations of TGF- $\beta 1$ (1754.690 $\pm 3416.487 \mathrm{pg} / \mathrm{ml})$ and IL-10 (2731.7101 \pm $6.1613 \mathrm{pg} / \mathrm{ml}$ ) were observed in A2780-conditioned than in control medium.

Conclusion: TGF- $\beta 1$ and IL-10 play pivotal roles in EOC and can lead to immune evasion. Targeting these cytokines for tumour treatment, specifically at early stages, may prevent tumour progression.
\end{abstract}

Keywords: Epithelial ovarian cancer, TGF- $\beta 1$, IL-10, histopathology

Tropical Journal of Pharmaceutical Research is indexed by Science Citation Index (SciSearch), Scopus, International Pharmaceutical Abstract, Chemical Abstracts, Embase, Index Copernicus, EBSCO, African Index Medicus, JournalSeek, Journal Citation Reports/Science Edition, Directory of Open Access Journals (DOAJ), African Journal Online, Bioline International, Open-J-Gate and Pharmacy Abstracts

\section{INTRODUCTION}

Ovarian cancer is a leading cause of death among gynaecological malignancies. Several surveys have been conducted to determine the causative factors in the development of malignant ovarian epithelial cells. Epithelial ovarian cancer (EOC) accounts for about 85-90 $\%$ of all ovarian cancers and is the leading cause of gynaecological malignancies in North America and worldwide. Despite all of the recent advances in treatment, the 5-year survival of patients with advanced stage disease remains less than $40 \%[1,2]$.

Ovarian cancer cells have the ability to create an immunosuppressive environment around the tumour and to escape immune monitoring. Ovarian cancer spreads intra-abdominally and is restricted primarily to the peritoneal cavity, where 
most immunosuppressive activity takes place, modulated by the tumour microenvironment. Hence, this microenvironment modulates invasion, progression, and metastasis of ovarian cancer. Taking place in the tumour microenvironment are cell-cell signalling and cellular-extracellular component interactions, which regulate inter/intracellular processes, such as proliferation, differentiation, and programmed cell death, as well as secretion and activation of soluble cytokines [3]. Despite the availability of diverse treatment strategies and accessibility to new cytotoxic agents, survival rates have not substantially improved [4]. Therefore, new treatment options targeting cancer cells and, in particular, putative cancer stem cells are required as first-line therapies and/or initial treatment of recurrent ovarian cancer.

The transforming growth factor $\beta$ (TGF- $\beta$ ) superfamily signalling pathways are ubiquitous and essential regulators of cellular processes, such as proliferation, differentiation and migration. TGF- $\beta$ signalling pathways also play essential roles in physiological processes, including embryonic growth, angiogenesis, and wound healing. Appropriate regulation of these pathways is needed, because either a deficiency or an excess of a specific TGF- $\beta$ superfamily ligand may result in pathology [5].

TGF- $\beta$ is a cytokine with pleiotropic functions in hematopoiesis, angiogenesis, cell proliferation, differentiation, migration, and apoptosis [6]. TGF$\beta$ also inhibits T-cell functions by blocking both proliferation and specialization. Despite the existence of tumour-specific antigens and the presence of tumour-specific immune cells, the majority of tumours manage to avoid immunemediated destruction. Several mechanisms have been proposed for tumour immune evasion. One such mechanism is believed to be mediated by TGF- $\beta 1$, an immunosuppressive cytokine found at the site of most tumours [8,9], and suggests that TGF- $\beta$ uses multiple mechanisms to ensure immunological tolerance, which is a critical feature of autoimmune and inflammatory disorders. TGF- $\beta 1$ is an essential regulator of immune responses, suppressing B-cell proliferation and synthesis of immunoglobulin (IgG and $\operatorname{lgM}$ ), and blocking proliferation of thymocytes, T-lymphocytes, and large granular lymphocytes [10-12]. Interleukin-10 (IL-10) is another immune regulatory cytokine that targets $\mathrm{T}$ cells, antigen-presenting cells, and related immune cells. Immunosuppressive roles of IL-10 in the tumour microenvironment have also been reported; however, some studies suggest immunostimulatory functions of $\mathrm{IL}-10$, and the role of IL-10 in ovarian cells is controversial.

In the present work, expression levels of TGF- $\beta 1$ and IL-10 were determined in ovarian tissues, including malignant, borderline, and benign tumour and normal tissues. Furthermore, correlation between expression levels of these cytokines and clinicopathologic features, and potential involvement of these cytokines in the immunosuppressive microenvironment of ovarian tumours were also determined.

\section{EXPERIMENTAL}

\section{Clinical specimens}

One hundred and twenty-five patients (20-60 years of age) with histologically confirmed primary ovarian tumours were classified in the histological groups malignant $(n=60)$, borderline $(n=15)$, and benign $(n=50)$. These specimens were obtained from patients undergoing primary debulking surgery at the Oncology Cancer Center Southern Medical University, Guangzhou, China. The malignant group included serous adenocarcinoma $(n=48)$, malignant mixed mesodermal tumours $(n=5)$, mucinous adenocarcinoma $(n=6)$, clear-cell carcinomas ( $n$ $=5)$, non-Brenner type transitional cell carcinoma $(n=3)$, and malignant Brenner tumour $(n=2)$. The borderline group consisted of serous $(n=6)$ and mucinous $(\mathrm{n}=10)$. The benign group included serous cystadenoma $(n=7)$, mucinous cystadenoma $(n=9)$, endometrial cyst $(n=3)$, simple cyst $(n=5)$, fibroma $(n=4)$, cystic follicle $(n=1)$, and serous cysts $(n=1)$. All experiments were performed following the protocols recommended by the Research Ethics Committees of the Department of Gynecologic, Oncology Cancer Center Southern Medical University, Guangzhou, China; Department of Gynecologic, Guangzhou Women and Children's Medical Center, Guangzhou Medical College, Guangzhou, China; Department of Gynecology and Obstetrics, Guangdong Provincial Corps Hospital, Chinese People Armed Forces, Guangzhou, China; Guangzhou Women and Children's Medical Center, Affiliate of Guangzhou Medical College, Guangzhou, China.

\section{Immunohistochemistry}

Ovarian tumour specimens free of necrotic tissue were identified from primary and metastatic tumours by a pathologist. Tissues were embedded in PolyFreeze tissue freezing medium (Polysciences, Warrington, PA, USA), snapfrozen in liquid nitrogen, and stored at $-80^{\circ} \mathrm{C}$. Use of frozen, rather than paraffin-embedded, 
specimens has the advantage that unmasking of denatured antigens is not needed. Serial $5-\mu \mathrm{m}$ sections of the tumours were excised, mounted on Superfrost Plus microscope slides, and airdried. Staining was carried out as previously described [13]. Briefly, the mounted sections were fixed in acetone for $5 \mathrm{~min}$ and rapidly transferred to phosphate-buffered saline (PBS). The slides were washed with $0.3 \% \mathrm{H}_{2} \mathrm{O}_{2}$ in methanol for $10 \mathrm{~min}$. After a short rinse, the slides were immersed in citrate buffer and heated at $100{ }^{\circ} \mathrm{C}$ in a water bath for 15-20 min. Slides were cooled to room temperature, rinsed, and normal goat serum was applied to the sections for $20 \mathrm{~min}$ to block nonspecific binding. Sections were then incubated overnight at $4{ }^{\circ} \mathrm{C}$ with specific antibodies directed against TGF- $\beta 1$ and IL-10 (Biolegend, San Diego, CA, US). Localization of antigen-antibody complexes was performed with the streptavidin-peroxidase technique using SP® Kits (Zymed, South San Francisco, CA, USA). Finally, sections were counterstained with Mayer's hematoxylin.

\section{Cells and culture}

A2780 human ovarian cancer cells were obtained from the American Type Culture Collection (Manassas, VA, USA), cultured in RPMI 1640 (Gibco-BRL/Life Technologies, Grand Island, NY, USA) containing $15 \%$ calf serum (Gibco-BRL/Life Technologies), $100 \mathrm{U} / \mathrm{ml}$ penicillin, and $100 \mathrm{mg} / \mathrm{ml}$ streptomycin, and maintained at $37^{\circ} \mathrm{C}$ in a humidified atmosphere of $5 \% \mathrm{CO}_{2}$. When cultures were 85-90\% confluent, the medium was replaced with serumfree RPMI 1640. The conditioned medium was decanted and used in further experiments. Concentrations of TGF- $\beta 1$ and $\mathrm{IL}-10$ in the conditioned medium were determined using a human enzyme-linked immunosorbent assay (ELISA) kit (R\&D Systems, Minneapolis, MN, USA). Concentrated serum-free RPMI 1640 served as negative control.

Peripheral blood mononuclear cells (PBMCs) from healthy adult donors who had provided consent were separated by Ficoll-Hypaque (GE Healthcare, Little Chalfont, UK) density gradient centrifugation. The plastic-adherence method was applied to separate monocytes from lymphocytes. Lymphocytes and monocytes were cultured in serum-free AIM-V medium, containing $1,000 \mathrm{U} / \mathrm{mL}$ recombinant human (Rh) granulocyte-macrophage colony stimulating factor (GM-CSF) and 1,000 U/mL Rh IL-4 (Peprotech) to generate immature dendritic cells, at $37^{\circ} \mathrm{C}$ in a humidified atmosphere of $5 \% \mathrm{CO}_{2}$ for 6 days. Then conditioned A2780 cell medium or negative control medium was added to the culture medium of lymphocytes and immature dendritic cells, and cells were incubated for another 3 days. Human TGF- $\beta 1$ and IL-10 were added to cultures of lymphocyte and immature DCs separately.

\section{Flow cytometric analysis}

Flow cytometric analysis was carried out using the method described by Liu et al [3]. Briefly, dendritic cells were labeled extracellularly with fluorescein isothiocyanate (FITC)-conjugated anti-human CD83 and phycoerythrin (PE)conjugated anti-human CD1a (BD Biosciences, San Jose, CA, USA). The cells were stained intracellularly with allophycocyanin (APC)conjugated anti-human FoxP3 (eBioscience, San Diego, CA, USA). FITC-conjugated normal mouse IgG1, PE-conjugated normal mouse IgG2, and APC-conjugated normal mouse IgG2 (BD Biosciences) were used as isotype controls. At least 50,000 cells/sample were processed with an LSR II cell analyser (BD Biosciences), and the data were analysed with DIVA software (BD Biosciences).

\section{Statistical analysis}

Kruskal-Wallis test was used to measure TGF- $\beta 1$ and IL-10 expression in ovarian tissues. The probability of survival was estimated by KaplanMeier and log rank test. Predictive factors were defined by multifactor logistic regression. $P<$ 0.05 was considered to be statistically significant. All statistical analyses were performed using GraphPad Prism 6.0 (GraphPad Software, La Jolla, CA, USA).

\section{RESULTS}

\section{TGF- $\beta 1$ and IL-10 expression in EOC patients}

The average photosensitive density of TGF- $\beta 1$ is $0.210-0.400$; therefore, intervals of 0.010 from 0.163 to 0.440 were calculated and analysed by Kaplan-Meier and log rank tests to define the cutoff level. The greatest difference occurred at $A=$ $0.351(p=0.013)$. The survival time of the TGF$\beta 1$ low group $(A<0.325)$ was $76.90 \pm 8.14$ months, whereas survival of the TGF- $\beta 1$ high was $65.0 \pm 7.98$ months. The difference between these groups approached statistical significance ( $p=0.048)$. Immunohistochemical analysis of TGF- $\beta 1$ and IL-10 was carried out in normal ovarian tissue samples and in samples of benign and malignant ovarian tumours (Figure 1). 


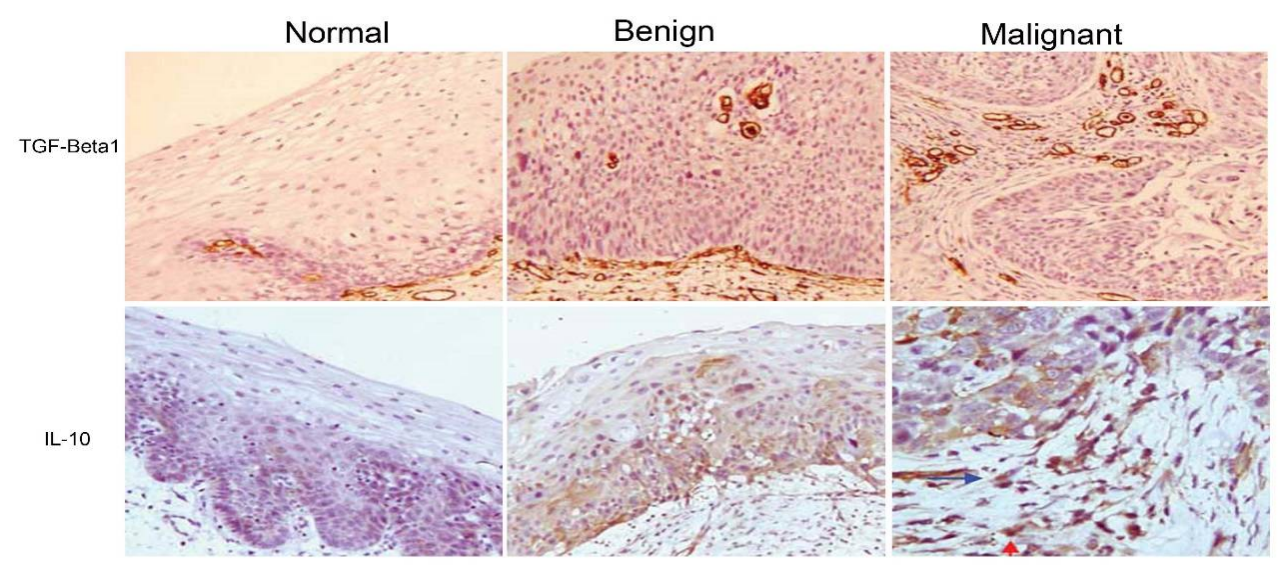

Figure 1: Immunohistochemical staining of TGF- $\beta 1$ and IL-10 in the indicated types of ovarian tissue specimens $(\times 200)$

TGF- $\beta 1$ was not expressed in normal ovarian tissue; it was weakly expressed in ovarian mucinous cystadenoma and ovarian borderline serous and markedly expressed in serous ovarian cancer. IL-10 was not expressed in normal ovarian tissue; it was weakly expressed in ovarian mucinous cystadenoma, moderately expressed in borderline serous cystadenoma, and significantly expressed in ovarian carcinoma.

IL-10 expression was observed in 45 of 79 specimens $(56.96 \%)$. The survival time of the negative group was $91.728 \pm 7217$ months, whereas that of the positive group (45/79) was $60.411 \pm 9.512$ months $(p=0.548)$ (Figure $2 \mathrm{~A}$ and $B)$.

\section{Clinical and pathological profiles and patient prognosis}

Clinicopathological factors including patient age, histological grade and FIGO stage of the tumour, residual tumour after surgery, lymphatic metastasis, and standard chemotherapy (data not shown) were examined. The results showed that TGF- $\beta 1$, residual tumour after surgery, and standard chemotherapy are prognostic factors for EOC ( $p=0.121,0.231$, and 0.121 , respectively). Correlation of TGF- $\beta 1$ expression with other clinicopathological factors was also recorded (data not shown). No substantial differences were observed in clinicopathological factors of TGF- $\beta 1$ Low and TGF- $\beta 1$ High, which implies that TGF- $\beta 1$ is an independent factor that does not influence the prognosis of EOCs.

\section{TGF- $\beta 1$ and IL-10 expression levels in culture supernatants}

High levels of TGF- $\beta 1$ (1754.690 \pm 3416.487 $\mathrm{pg} / \mathrm{ml})$ and IL-10 (2731.7101 $\pm 6.1613 \mathrm{pg} / \mathrm{ml})$ were detected in culture medium conditioned by A2780 cells compared to unconditioned negative control medium.

\section{Ovarian cancer immunosuppressive responses}

Different concentrations of A2780 conditioned medium were added to lymphocyte and immature dendritic cell (DC) cultures. When the concentration of TGF- $\beta 1$ in the conditioned medium was high (final concentration $>300 \mathrm{pg} / \mathrm{ml}$ in $\mathrm{CF}^{\mathrm{High}}$ ), the proportion of immature $\mathrm{CD} \mathrm{a}^{+}$ immature DCs increased significantly, whereas the proportion of $\mathrm{CD} 3^{+}$mature DCs decreased. These responses were not induced by treatment of cells with the same concentration of TGF- $\beta 1$ in the absence of A2780 conditioned medium. When inadequate supernatant $\left(\mathrm{CF}^{\mathrm{Low}}+\mathrm{TGF}-\beta 1\right.$ or $\left.\mathrm{CF}^{\text {Low }}+\mathrm{IL}-10\right)$ was incubated with lymphocytes and DCs, DC maturation was impaired. Furthermore, ELISA was performed to determine IL-10 concentration in DC cultures and showed that DCs cultured in $\mathrm{CF}^{\mathrm{High}}$ secreted higher levels of IL-10 than those cultured in CF ${ }^{\text {Low }}$. It was also observed that the presence of enough TGF- $\beta 1$ in supernatant interfered with dendritic cell maturation (Figure $2 \mathrm{C} \& \mathrm{D}$ ).

\section{Flow cytometry data}

Various amounts of A2780 conditioned medium were added to DC and lymphocyte cultures, and maturation and regulation of T-lymphocytes were analysed using flow cytometry (Figure 3 ). When cells were treated with $\mathrm{CF}^{\text {High }}$ (final TGF- $\beta 1$ concentration $>800 \mathrm{pg} / \mathrm{ml}$ ), the number of mature $\mathrm{CD}^{+} 3^{+} \mathrm{DCs}$ decreased, and CD1a expression significantly increased.

Trop J Pharm Res, December 2015; 14(12): 2182 
(A)
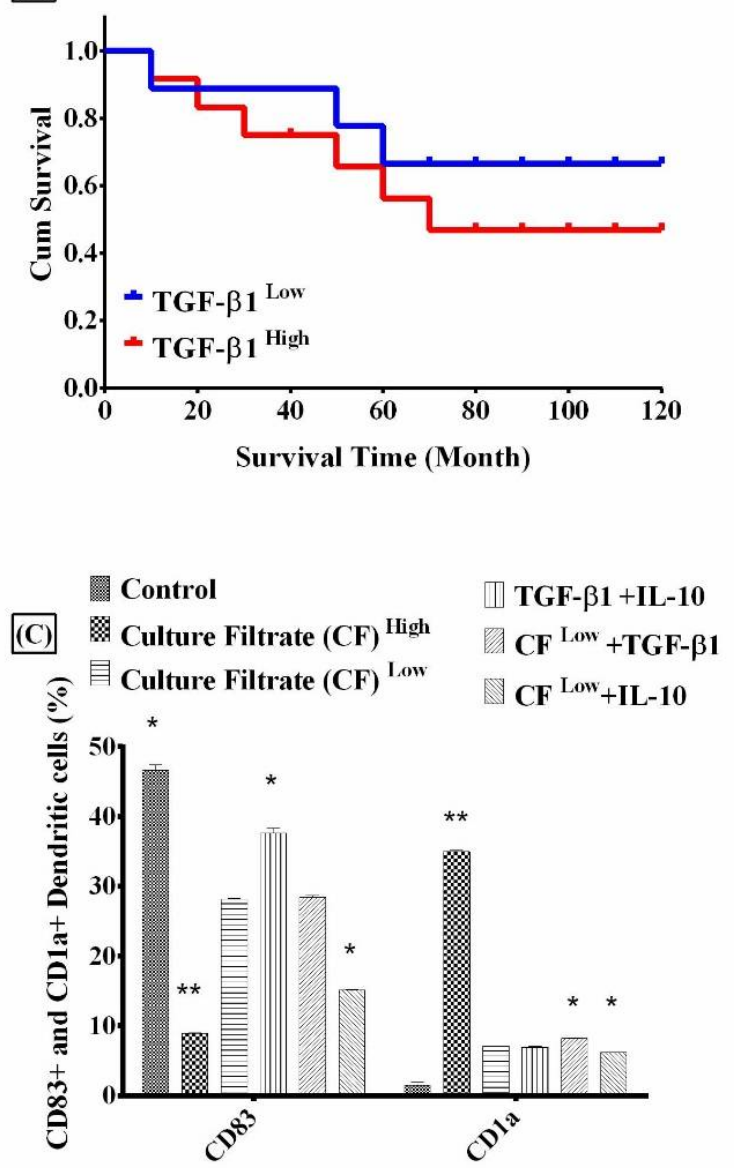

(B)
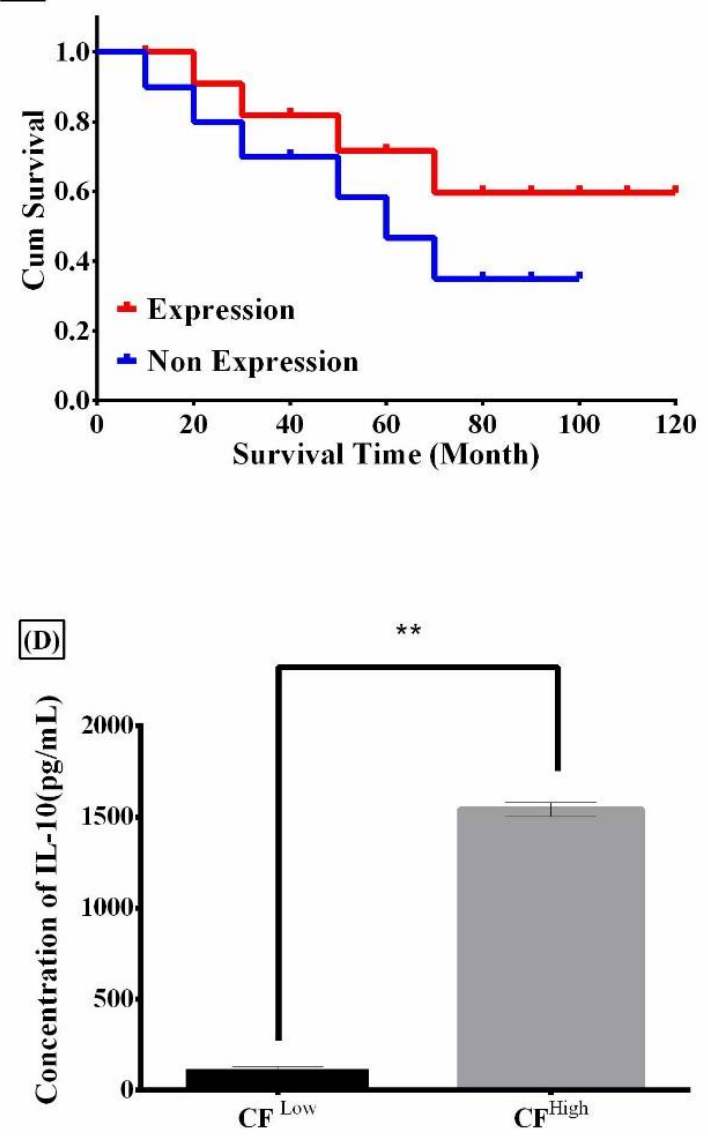

Figure 2: (A). Survival curve for 100 epithelial ovarian cancer (EOC) patients with low or high level of TGF- $\beta 1$ expression. (B). Survival curve of $100 \mathrm{EOC}$ patients with IL-10 nonexpression. (C) Immunosuppressive response of CD83+ and CD1a+ dendritic cells (DCs) to the indicated treatments (" $p<0.01, " p<0.05)$.(D). Concentration of IL-10 in DC culture supernatants (" $p<0.01)$. Cum, cumulative

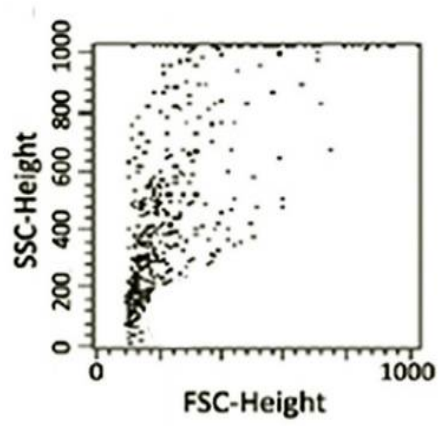

Figure 3: Flow cytometric analysis of immunosuppressive response. Dendritic cells were gated in R1, and CD83 ${ }^{+}$ and $\mathrm{CD}_{1} \mathrm{a}^{+}$dendritic cells were counted

\section{DISCUSSION}

EOC is the most lethal disease among gynaecological malignancies. It is the fourth leading cause of malignant neoplastic diseaseassociated deaths in women in the United States and the fifth most common malignancy in adult females in developed countries [14]. TGF- $\beta 1$ is a multifunctional growth factor that has profound regulatory effects on many developmental and physiological processes. Hurteau et al [17] identified TGF- $\beta$ in most ovarian tumour tissue samples examined, whether benign or malignant. Some published work has also reported the contradictory observation that not all ovarian cancers express TGF- $\beta$. 
In the majority of patients, risk of EOC is linked to incessant ovulation, i.e., chronically repeated formation of stromal epithelial clefts and inclusion cysts after ovulation [19]. Members of the TGF- $\beta$ family have shown distinct cell-specific patterns of expression at various stages of development, including in the three primary embryonic germ layers [20]. The TGF- $\beta$ family is an important family of cytokines that may promote tumour development in vivo through several mechanisms, including interference with antitumour T-cell immune responses, alteration of factors in the stroma and extracellular matrix, and promotion of angiogenesis. TGF- $\beta$ isotypes have been found in malignant tumour and normal ovarian tissues [21]. EOC is notoriously lethal and little is known about its pathogenesis [22].

In the present study, roles played by TGF- $\beta 1$ and IL-10 in the initiation and spread of EOC were determined. Samples of normal and tumour tissue were collected from randomly selected ovarian cancer patients, and relative levels of TGF- $\beta 1$ and IL- 10 expression were determined using immunohistochemical staining of tissue sections. TGF- $\beta 1$ and IL-10 expression were significantly higher in cancerous tissues than in normal tissues. These results confirmed that these cytokines are frequently overexpressed in EOC. Some studies had suggested that expression of these cytokines is correlated; e.g., TGF- $\beta 1$ was simultaneously overexpressed with IL-10 and vascular endothelial growth factor (VEGF) in oesophageal squamous cell carcinoma, and IL-10 expression was induced in melanoma. In contrast to those findings, TGF- $\beta 1$ and IL-10 expression were not correlated in the present study. Statistical analysis revealed that TGF- $\beta 1$ expression, residual tumour, and chemotherapy were important prognostic factors for EOC. Moreover, no correlation was observed between TGF- $\beta 1$ expression, FIGO stage, patient age, residual tumour, histological grade, lymphatic metastasis, or standard chemotherapy. Therefore, TGF- $\beta 1$ may be considered an independent prognostic factor for EOC.

TGF- $\beta 1$ and IL-10 are important cytokines that have been reported to induce immunosuppression, and overexpression of the cytokines suggests their possible involvement in immune evasion by EOC. DCs are considered the most important antigen-presenting cells. Based on the role of DCs in immunosuppressive responses, immunosuppressive functions of IL10 and TGF- $\beta 1$ were probed. In vitro experiments revealed that TGF- $\beta 1$ interfered with DC maturation, and immature DCs secreted increased levels of IL-10. Although TGF- $\beta 1$ and IL-10 play important roles in immunosuppressive responses, the cytokines alone were not able to elicit these responses and, in our study, treatment of cells with A2780 conditioned medium was required to induce immunosuppression.

\section{CONCLUSION}

This study confirmed that overexpression of TGF- $\beta 1$ and IL-10 performs a significant function in EOC and contributes to frequent immune evasion events. Targeting these cytokines for tumour treatment, particularly in the early stages of disease, might prevent tumour progression to advanced stages.

\section{ACKNOWLEDGEMENT}

This work was supported by Guangdong Natural Science Foundation (No. S2013010016011).

\section{REFERENCES}

1. Hunn J, Rodriguez GC. Ovarian cancer: etiology, risk factors, and epidemiology. Clin Obstet Gynecol 2012; 55: 3-23.

2. Labrie M, Vladoiu MC, Grosset AA, Gaboury L, St-Pierre $Y$. Expression and functions of galectin-7 in ovarian cancer. Oncotarget 2014; 5: 7705-7721.

3. Liu CZ, Zhang L, Chang XH, Cheng YX, Cheng HY, Ye $X$, Fu TY, Chen J, Cui H. Overexpression and immunosuppressive functions of transforming growth factor 1, vascular endothelial growth factor and interleukin-10 in epithelial ovarian cancer. Chin $J$ Cancer Res 2012; 24: 130-137.

4. Kakar SS, Ratejczak MZ, Powell KS, Moghadamfalahi M, Miller DM, Batra SK, Singh SK. Withaferin a alone and in combination with Cisplatin suppresses growth and metastasis of ovarian cancer by targeting putative cancer stem cells. PloS one 2014; 9: e107596.

5. Gordon KJ, Blobe GC. Role of transforming growth factor- $\beta$ superfamily signaling pathways in human disease. BBA-Mol Basis Dis 2008; 1782:197-228.

6. Gonzalo-Gil E, Galindo-Izquierdo $M$. Role of Transforming Growth Factor-Beta (TGF) Beta in the Physiopathology of Rheumatoid Arthritis. Reumatol Clin 2014; 10:174-179.

7. Gorelik L, Constant S, Flavell RA. Mechanism of Transforming Growth Factor $\beta$-induced Inhibition of $T$ Helper Type 1 Differentiation. J Exp Med 2002; 195:1499-1505.

8. Gorelik L, Flavell RA. Immune-mediated eradication of tumour s through the blockade of transforming growth factor-[beta] signaling in $T$ cells. Nat Med 2001; 7:1118-1122.

9. Kriegel MA, Li MO, Sanjabi S, Wan YY, Flavell RA. Transforming growth factor-beta: recent advances on

Trop J Pharm Res, December 2015; 14(12): 2184 
its role in immune tolerance. Curr Rheumatol Rep 2006; 8: 138-144.

10. Kehrl JH, Roberts $A B$, Wakefield $L M$, Jakowlew S, Sporn $M B$, Fauci AS. Transforming growth factor beta is an important immunomodulatory protein for human $B$ lymphocytes. J Immunol 1986; 137: 3855-3860.

11. Kehrl JH, Thevenin C, Rieckmann P, Fauci AS. Transforming growth factor-beta suppresses human $B$ lymphocyte Ig production by inhibiting synthesis and the switch from the membrane form to the secreted form of Ig mRNA. J Immunol 1991; 146 . 4016-4023

12. Ortaldo JR, Mason AT, O'Shea JJ, Smyth MJ, Falk $L A$, Kennedy IC, Longo DL, Ruscetti FW. Mechanistic studies of transforming growth factor-beta inhibition of IL-2-dependent activation of CD3- large granular lymphocyte functions. Regulation of IL-2R beta (p75) signal transduction. J Immunol 1991; 146: 3791 3798.

13. Zhang HZ, Ordonez NG, Batsakis JG, Chan JC. Monoclonal antibody recognizing a carcinoembryonic antigen epitope differentially expressed in human colonic carcinoma versus normal adult colon tissues. Cancer Res 1989; 49: 5766-5773.

14. Jemal A, Bray F, Center MM, Ferlay J, Ward E, Forman D. Global cancer statistics. CA: A Cancer Journal for Clinicians 2011; 61: 69-90.

15. Shull MM, Ormsby L, Kier AB, Pawlowski S, Diebold RJ, Yin M, Allen R, Sidman C, Proetzel G, Calvin D, et al. Targeted disruption of the mouse transforming growth factor- $\beta 1$ gene results in multifocal inflammatory disease. Nature 1992; 359: 693-699
16. Batchu RB, Gruzdyn OV, Moreno-Bost AM, Szmania S, Jayandharan G, Srivastava A, Kolli BK, Weaver DW, van Rhee F, Gruber SA. Efficient lysis of epithelial ovarian cancer cells by MAGE-A3-induced cytotoxic $T$ lymphocytes using rAAV-6 capsid mutant vector. Vaccine 2014; 32:938-943.

17. Hurteau J, Rodriguez GC, Whitaker RS, Shah S, Mills G, Bast RC, Berchuck A. Transforming growth factor-beta inhibits proliferation of human ovarian cancer cells obtained from ascites. Cancer 1994; 74 : 93-99.

18. Berchuck A, Rodriguez G, Olt G, Whitaker R, Boente MP, Arrick BA, Clarke-Pearson DL, Bast RC Jr. Regulation of growth of normal ovarian epithelial cells andovarian cancer cell lines by transforming growth factor-B. Am J Obstet Gynecol 1992; 166: 676-684.

19. Risch HA. Hormonal etiology of epithelial ovarian cancer, with a hypothesis concerning the role of androgens and progesterone. I National Cancer I 1998; 90:1774-1786.

20. Pelton RW, Saxena B, Jones M, Moses HL, Gold LI. Immunohistochemical localization of TGF beta 1, TGF beta 2, and TGF beta 3 in the mouse embryo: expression patterns suggest multiple roles during embryonic development. J Cell Biol 1991; 115: 1091 1105.

21. Gordinier ME, Zhang $H Z$, Patenia R, Levy LB, Atkinson EN, Nash MA, Katz RL, Platsoucas $C D$, Freedman RS. Quantitative analysis of transforming growth factor beta 1 and 2 in ovarian carcinoma. Clin Cancer Res 1999; 5: 2498-2505

22. Shan W, Liu J. Epithelial ovarian cancer. Cell Cycle 2009; 8:731-735. 